\title{
Intergrowth versus fenton: há diferença no diagnóstico de retardo de crescimento extrauterino em prematuros durante internamento hospitalar?
}

\author{
Intergrowth versus fenton: is there a difference in the diagnosis of extrauterine growth \\ retardation in preterm infants during hospitalization?
}

\author{
Louise Perna Martins da Cunha ${ }^{1 *}$, Ana Cecília Travassos Santiago ${ }^{2}$, Crésio de Aragão Dantas Alves $^{3}$ \\ ${ }^{1}$ Nutricionista, doutoranda em Processos Interativos dos Órgãos e Sistemas, Instituto de Ciências da Saúde-UFBA. \\ ${ }^{2}$ Pediatra Neonatologista, Professora Associada de Pediatria - UFBA. ${ }^{3}$ Endocrinologista Pediátrico, Professor Associado \\ de Pediatria - UFBA.
}

\begin{abstract}
Resumo
Introdução: alterações no padrão de crescimento de recém-nascidos prematuros podem ter implicações para sua saúde futura. A literatura dispõe de diversas ferramentas e pontos de corte para avaliação da sua adequação, logo, diferentes diagnósticos podem ser obtidos a depender do parâmetro adotado. Objetivo: determinar a diferença no diagnóstico de Retardo de Crescimento Extrauterino em prematuros, durante internamento hospitalar, conforme as curvas de Fenton e Intergrowth. Metodologia: trata-se de estudo transversal, com dados secundários, coletados durante o internamento em unidade de terapia intensiva e de cuidados intermediários convencionais neonatais de uma maternidade pública, em 2019, Coletaram-se medidas de peso e perímetro cefálico ao nascer e no momento da alta/transferência e calcularam-se seus respectivos indicadores antropométricos de acordo com as duas curvas. Utilizaramse duas classificações para o Retardo: diagnóstico de Pequeno para Idade Gestacional na alta/transferência; queda no escore Z dos indicadores maior ou igual a 1 entre o nascimento e a alta/transferência. Resultados: Não houve diferença em relação ao número de crianças classificadas como Pequeno para Idade Gestacional ao nascer, entre as curvas. Porém, no momento da alta/transferência houve maior prevalência de Pequeno para a Idade Gestacional/Retardo de Crescimento Extrauterino, de acordo com Fenton $(73,6 \%$ versus 64,9\%). A análise longitudinal dos indicadores de crescimento para caracterização do referido retardo por meio da curva de Fenton também detectou maior número de diagnósticos. Conclusão: conclui-se que o diagnóstico do retardo apresentou diferenças entre os referenciais. Os parâmetros de Fenton determinaram maior ocorrência dele no momento do desfecho, independente da realização da avaliação transversal ou longitudinal dos indicadores.

Palavras-chave: Crescimento. Desenvolvimento infantil. Recém-nascido prematuro. Doenças do prematuro. Recém-nascido de baixo peso.
\end{abstract}

\begin{abstract}
Introduction: changes in growth pattern of preterm infants may have implications for their future health. Literature has several tools and cutoff points to assess its adequacy, therefore, different diagnosis may be obtained depending on the adopted parameter. Objective: determine the difference in diagnosis of extrauterine growth restriction (EUGR) in preterm infants during hospitalization based on Fenton (2013) and Intergrowth-21 (2014) curves. Methods: this is a cross-sectional study with secondary data which were collected during hospitalization in an intensive care unit and neonatal conventional intermediate care in a public maternity hospital, in 2019. Results: weight and head circumference measurements were collected at birth and at discharge/transfer and their respective anthropometric indicators were calculated according to Fenton and Intergrowth-21 curves. The following EUGR criteria were used: diagnosis of small for gestational age (SGA) at discharge/transfer; decrease in Z score for indicators higher or equal to 1 between birth and discharge/transfer. There was no difference in the number of children classified as SGA at birth between the curves. However, at the time of discharge/ transfer there was a higher prevalence of SGA/EUGR according to Fenton (73.6\% versus 64.9\%). Longitudinal analysis of growth indicators for EUGR using Fenton curve also detected a higher number of patients with EUGR. Conclusion: the conclusion is that EUGR diagnosis showed differences between Fenton and Intergrowth methods. Fenton's parameters determined a higher occurrence of EUGR at the time of outcome, regardless of whether indicators were cross-sectionally or longitudinally evaluated.
\end{abstract}

Keywords: Growth. Child development. Premature infant. Premature infant diseases. Low birth weight infant.

\section{INTRODUÇÃO}

Anormalidades no padrão de crescimento de prematuros podem acarretar em implicações futuras (REDDY et al., 2021). Embora existam várias formas de avaliar o

Correspondente/Corresponding: *Louise Perna Martins da Cunha Instituto de Ciências da Saúde da Universidade Federal da Bahia - End: Av. Reitor Miguel Calmon, $\mathrm{s} / \mathrm{n}$ - Vale do Canela - 40.231-300 Salvador/ BA - Tel: (71) 98543-8330-E-mail: loupernamdc@gmail.com crescimento pós-natal dessas crianças, não há um consenso internacional sobre qual deveria ser seu padrão normal e como monitorá-lo (REDDY et al., 2021). A multiplicidade de formas e de ferramentas de avaliação faz com que haja divergências entre os diagnósticos, dificultando a comparação dos dados e prejudicando a avaliação epidemiológica e seus resultados de importância clínica para o seguimento dessas crianças. 
Retardo do crescimento extrauterino (RCEU) tem sido relatado como muito prevalente, mesmo nos países industrializados (HENRIKSEN et al., 2009), o que leva ao questionamento sobre a real acurácia das avaliações feitas por meio das ferramentas disponíveis e sobre a eficácia das terapias e diretrizes terapêuticas do cuidado dos recém-nascidos pré-termos (CLEMINSON; ZALEWSKI; EMBLETON, 2017).

Seu diagnóstico é feito por meio da análise do crescimento pós-natal, utilizando-se as curvas de crescimento. Para os prematuros (PT), estão disponíveis curvas de crescimento fetal, consideradas de referência, a exemplo da curva de Fenton (FENTON; KIM, 2013); e as curvas de crescimento pós-natal do PT, como a curva do Intergrowth (VILLAR, J et al., 2014), considerada prescritiva para o PT saudável. A análise do trajeto da curva ou simplesmente um ponto em dado momento poderá determinar sua ocorrência (CORDOVA; BELFORT, 2020).

A ocorrência do retardo do crescimento, seja ele intra ou extrauterino, está associada a maior mortalidade e comprometimento do neurodesenvolvimento em longo prazo (ONG et al., 2015), de modo que a gravidade dessa restrição está associada ao maior risco de morbidade futura (EHRENKRANZ, 2007).

Sendo assim, é fundamental avaliar as curvas atualmente disponíveis para avaliação antropométrica do recém-nascido PT ao nascer e no seu seguimento, as limitações e vantagens de cada uma, o desempenho em determinar a ocorrência de RCEU, diante de diferentes populações e condições assistenciais. Com base nesses dados, o presente trabalho objetiva determinar a diferença no diagnóstico de RCEU em PT durante internamento hospitalar, conforme as curvas de Fenton (FENTON; KIM, 2013) e Intergrowth (VILLAR et al., 2015).

\section{METODOLOGIA}

Trata-se de um estudo de corte transversal, avaliando dados secundários obtidos da base de dados QualiNeo, do Ministério da Saúde do Brasil, de pacientes admitidos na unidade de terapia intensiva neonatal (UTIN) e unidade de cuidados intermediários convencionais (UCINCO) de uma maternidade pública na cidade de Salvador, no ano de 2019. Os dados foram coletados e registrados por meio de formulários estruturados, elaborados pelo Ministério da Saúde e preenchidos pela equipe assistencial ao longo do internamento hospitalar. Tal iniciativa objetiva apoiar maternidades que ainda apresentam elevadas taxas de mortalidade neonatal para qualificação das práticas de gestão e atenção ao recém-nascido (RN), por meio de treinamentos e ações voltadas à conscientização dos profissionais, além do monitoramento das práticas assistenciais.

Os dados antenatais incluíram: uso de esteroide, tempo de bolsa rota, tipo de parto, reanimação na sala de parto, escore de APGAR e idade gestacional. Coletaram-se ainda dados sobre a terapia nutricional e informações sobre o curso clínico durante o internamento
Foram incluídos os $\mathrm{RN}$ admitidos durante o ano de 2019. Excluíram-se os pacientes nascidos a termo, com idade gestacional maior ou igual a 37 semanas (68), os que não apresentaram registro de idade gestacional no formulário (2), paciente cujo sexo foi indeterminado (1) e pacientes que evoluíram a óbito durante o internamento (11).

A idade gestacional (IG) foi determinada por meio do cálculo da data da última menstruação, ultrassom do primeiro trimestre e, na ausência dessa informações, por meio da estimativa pelo método de Capurro ou Ballard. A idade pós-menstrual e pós-concepcional foi obtida por meio da soma da idade gestacional à idade cronológica em semanas.

Coletaram-se as medidas do peso e do perímetro cefálico ao nascer e no momento do desfecho - alta/transferência para outra unidade -, contemplando portanto, a avaliação durante o tempo em que o paciente esteve internado na maternidade e calculando os indicadores antropométricos peso e perímetro cefálico para idade de acordo com a curva de Fenton (FENTON; KIM, 2013) e do Intergrowth (VILLAR et al., 2015), nesses dois momentos, tanto em percentis como escore $Z$.

Os percentis e escores $Z$ das medidas do nascimento e de alta foram calculados eletronicamente. Para os indicadores de Fenton, utilizou-se planilha de cálculo disponível em: https://ucalgary.ca/resource/preterm-growth-chart/calculators. Para os indicadores do Intergrowth, utilizaram-se duas curvas diferentes, uma para o análise ao nascimento e outra para o seguimento. Para cálculo dos percentis e escores $Z$ das medidas do nascimento, segundo Intergrowth, utilizou-se o software disponivel em http://intergrowth21. ndog.ox.ac.uk/. E, para análise das medidas na alta/transferência de acordo com esse mesmo padrão, utilizou-se outro software, disponível em http://intergrowth21.ndog. ox.ac.uk/preterm.

Os pacientes foram classificados em relação à adequação do peso para idade gestacional ao nascimento e no momento da alta ou transferência em pequenos (menor que o percentil 10), adequados (entre o percentil 10 e 90) e grandes para idade gestacional (maior que o percentil 90), PIG, AIG e GIG, respectivamente (VILLAR et al., 2014). Considerou-se como RCEU o diagnóstico de PIG no momento da alta/transferência, caracterizando diagnóstico transversal dessa condição (CORDOVA; BELFORT, 2020).

Como forma alternativa de diagnóstico do RCEU, calculou-se o delta entre os indicadores ao nascer e os apresentados no momento da alta hospitalar/ transferência em escore Z. Foi considerado como RCEU uma queda nos indicadores, cuja diferença tenha sido maior ou igual a 1 entre esses dois momentos, o que caracterizou diagnóstico longitudinal dessa condição (CORDOVA; BELFORT, 2020).

$O$ banco de dados foi construído e analisado no programa Social Package for Social Sciences (SPSS versão 20.0). A normalidade das variáveis contínuas foi avaliada por meio de estatística descritiva e testes de normalidade (Shapiro-Wilk). As variáveis não apresentaram distribuição semeIhante à curva normal ao nível de $5 \%$ e foram expressas por mediana e percentis 25 e 75 . Foi realizada a comparação 
das medianas das medidas e indicadores de crescimento entre os grupos PIG, de acordo com cada uma das curvas no momento do nascimento e da alta/ transferência, por meio do teste da soma dos postos sinalizados de Wilcoxon. Para avaliar a existência de diferença nas proporções de diagnósticos de nascimentos de crianças PIG (peso para idade gestacional ao nascimento $<$ P10) e a ocorrência de RCEU (delta entre o indicador de peso ao nascer e no momento da alta hospitalar/ transferência em escore $Z \geq 1$ ), entre as curvas de Fenton e Intergrowth, utilizou-se o teste de McNemar. O nível de significância adotado foi de $5 \%$.

O projeto foi aprovado no Comitê de Ética em Pesquisa da Maternidade Climério de Oliveira, pelo Parecer n. 4961843.

\section{RESULTADOS}

Fizeram parte do estudo 94 RN prematuros, $48,9 \%$ eram do sexo feminino sendo $69,1 \%$ (65) nascidos na própria unidade. A maioria dos pacientes incluídos nasceu de parto cesáreo $(64,68,1 \%)$ e não precisaram de reanimação na sala de parto $(46,48,9 \%)$.

O motivo do internamento incluiu causas variadas, sendo a mais comum o desconforto respiratório ou taquipneia transitória do RN e a hiperbilirrubinemia. A mediana do tempo de internamento foi de 40 dias $(18,5 ; 66)$. De um total de 88 pacientes, $21(23,8 \%)$ necessitaram de suporte ventilatório invasivo. Quanto aos pacientes analisados em relação ao uso do suporte não invasivo (87), 45 (51,7\%) necessitaram de CPAP durante o internamento. A mediana de tempo de uso de oxigênio, avaliada em 43 pacientes, foi de 23 dias $(1 ; 18)$.

Quatro pacientes $(4,3 \%)$ foram diagnosticados com hemorragia intracraniana (HIC) e o mesmo número de diagnósticos foi obtido para enterocolite necrotizante. Houve infecção precoce em 40 pacientes $(42,6 \%)$ e infecção tardia em 20 (21,3\%). Antibioticoterapia foi utilizada por 33 crianças $(35,1 \%)$ nas primeiras 48 horas de vida; e por $18(19,1 \%)$, até o 7 o dia de vida; 34 pacientes $(36,2 \%)$ não necessitaram de antibioticoterapia.

Dados sobre nutrição parenteral (NP) foram registrados em $47,9 \%$ dos pacientes, dos quais $71,1 \%$ tiveram a NP iniciada nas primeiras $24 \mathrm{~h}$ de vida e finalizada até o 14 을.

A maioria dos pacientes $(98,9 \%)$ usou nutrição enteral (NE). Mais da metade $(67,4 \%)$ iniciou o uso precocemente (nas primeiras 48 horas de vida) e 84,3\% até o 40 dia de vida. O principal leite ofertado como forma de primeira dieta foi o leite humano pasteurizado $(51,1 \%)$, seguido pelo leite da própria mãe cru $(35,1 \%)$. Percentual semelhante de uso de leite humano também foi observado no momento da alta/ transferência, em que $57,4 \%$ dos pacientes recebiam leite materno e $21,3 \%$ recebiam leite materno + fórmula infantil.

A Tabela 1 apresenta a idade gestacional, as medidas e os indicadores antropométricos ao nascer e suas classificações em PIG e AIG, de acordo com o referencial do Intergrowth e Fenton. As medianas das medidas apresentadas pelos pacientes do grupo PIG, de acordo com
Intergrowth, foram menores que as dos PIG, de acordo com Fenton, porém, os indicadores, tanto em escore $Z$ quanto em percentil, apresentaram comportamento contrário. Não houve, contudo, diferença estatística entre tais medianas, com exceção do perímetro cefálico para idade expresso em escore $Z$, de acordo com a curva de Fenton, que se apresentou menor para as crianças classificadas como PIG ao nascer por esta mesma referência (-1,06 por Fenton versus $-0,63$ por Intergrowth).

A Tabela 2 apresenta as mesmas variáveis da Tabela 1 no momento do desfecho. Observa-se aqui o oposto do visto ao nascimento: os nascidos PIG de acordo com Intergrowth foram maiores e mais pesados, com exceção para medida do perímetro cefálico. Os indicadores se comportaram da mesma forma: maiores de acordo com Intergrowth, com exceção do perímetro cefálico. Não houve contudo diferença estatística entre tais medianas.

$\mathrm{O}$ número de crianças diagnosticadas como GIG foi pequeno em ambos os grupos ( 3 de acordo com Fenton e 1 por Intergrowth), portanto, esses dados não foram incluídos nas referidas tabelas.

A frequência de nascimentos de crianças PIG entre os grupos foi muito semelhante, $26(27,7 \%)$ para o grupo Intergrowth e $24(25,5 \%)$ para o grupo Fenton, alcançando diferença um pouco maior na alta, caracterizando o diagnóstico transversal de RCEU, em 61 (64,9\%) e $67(73,6 \%)$, respectivamente. Ao nascimento não foi observada diferença estatística em relação à proporção dos diagnósticos de crianças PIG ao nascer, entre as classificações de Fenton e Intergrowth $(p=0,15)$. Porém, a proporção de crianças classificadas como PIG na alta por Fenton e Intergrowth foi estatisticamente diferente, com maior proporção de diagnósticos de acordo com a curva de Fenton $(p=0,00)$.

Quando analisados apenas os nascidos com menos de 33 semanas gestacionais (46), o resultado obtido foi o mesmo, com nascimento de 11 (23,9\%) PIG de acordo com Intergrowth e $9(19,6 \%)$ de acordo com Fenton, alcançando $34(73,9 \%)$ e $36(81,8 \%)$, respectivamente, no momento da alta/ transferência.

O número de diagnósticos concordantes para a análise transversal dos indicadores entre as curvas de Fenton e Intergrowth foi de 24 e 58 ao nascimento e na alta/transferência respectivamente.

Quanto à trajetória das curvas de peso para idade gestacional apresentada pelos pacientes avaliados, nota-se maioria expressiva de indicadores descendentes para mais de $90 \%$ dos pacientes, de acordo com ambas as curvas.

A mediana do delta entre os indicadores do nascimento e momento da alta/ transferência para os pacientes que apresentaram traçado descendente, de acordo com a curva do Intergrowth, foi de 1,21, variando de 0,01 a 5,81 e de 1,53 , e variando de 0,06 a 4,02 para a curva de Fenton.

Quando classificados em relação à ocorrência de RCEU por meio do diagnóstico longitudinal dessa condição (delta entre indicadores do nascimento e momento do desfecho maior que 1,0$)$, foi possível diagnosticar $64(70,3 \%)$ casos 
por meio da curva de Fenton e $51(64,2 \%)$, por Intergrowth. dos diagnósticos $(p=0,01)$. Foi observada diferença estatística em relação à proporção

Tabela 1 - Características antropométricas ao nascimento de lactentes nascidos PIG e AIG de acordo com os parâmetros de Intergrowth e Fenton.

\begin{tabular}{|c|c|c|c|c|c|c|c|c|}
\hline & \multicolumn{4}{|c|}{ Intergrowth } & \multicolumn{4}{|c|}{ Fenton } \\
\hline & \multicolumn{2}{|c|}{ PIG } & \multicolumn{2}{|c|}{ AIG } & \multicolumn{2}{|c|}{ PIG } & \multicolumn{2}{|c|}{ AIG } \\
\hline & Mediana & P25-75 & Mediana & P25-75 & Mediana & P25-75 & Mediana & P25-75 \\
\hline IG, semanas & 33,80 & $(30,00 ; 35,00)$ & 32,50 & $(29,6 ; 34,00)$ & 33,90 & $(30,40 ; 35,3)$ & 32,10 & $(29,6 ; 34,00)$ \\
\hline Peso, $\mathrm{g}$ & 1230,00 & $\begin{array}{r}(896 ; \\
1764,00)\end{array}$ & 1720,00 & $\begin{array}{r}(1300,00 \\
2153,00)\end{array}$ & 1435,00 & $\begin{array}{r}(918,00 \\
1776,00)\end{array}$ & 1680,00 & $\begin{array}{r}(1275,00 \\
2090,00)\end{array}$ \\
\hline $\mathrm{PC}, \mathrm{cm}$ & 29,00 & $(26,00 ; 31,00)$ & 30,00 & $(27,00 ; 32,00)$ & 29,00 & $(26,00 ; 31,00)$ & 30,00 & $(27,00 ; 32,00)$ \\
\hline $\mathrm{P} / \mathrm{I}$, Intergrowth, escore Z & $-1,71$ & $(-2,32 ;-1,47)$ & $-0,20$ & $(-0,51 ; 0,45)$ & $-1,73$ & $(-2,40 ;-1,49)$ & $-0,20$ & $(-0,64 ; 0,47)$ \\
\hline $\mathrm{P} / \mathrm{I}$, Intergrowth, percentil & 4,30 & $(1,00 ; 7,00)$ & 42,30 & $(30,70 ; 67,30)$ & 4,10 & $(0,90 ; 6,80)$ & 42,00 & $(26,30 ; 68,20)$ \\
\hline PC/I, Intergrowth, escore Z & $-1,20$ & $(-1,60 ;-0,80)$ & 0,00 & $(-0,70 ; 0,70)$ & $-1,18$ & $(-1,62 ;-0,81)$ & $-0,03$ & $(-0,73 ; 0,63)$ \\
\hline $\mathrm{PC} / \mathrm{I}$, Intergrowth, percentil & 12,00 & $(5,30 ; 22,2)$ & 49,50 & $(23,30 ; 76,10)$ & 12,00 & $(5,3 ; 21,00)$ & 48,90 & $(23,30 ; 73,50)$ \\
\hline$P / I$, Fenton, escore $Z$ & $-1,58$ & $(-1,89 ;-1,40)$ & $-0,26$ & $(-0,73 ; 0,13)$ & $-1,60$ & $(-1,94 ;-1,43)$ & $-0,26$ & $(-0,76 ; 0,15)$ \\
\hline $\mathrm{P} / \mathrm{I}$, Fenton, percentil & 5,73 & $(2,92 ; 8,05)$ & 39,91 & $(23,27 ; 55,01)$ & 5,49 & $(2,65 ; 7,63)$ & 39,75 & $(22,41 ; 55,88)$ \\
\hline $\mathrm{PC} / \mathrm{I}$, Fenton, escore $\mathrm{Z}^{*}$ & $-1,02$ & $(-1,39 ;-0,61)$ & $-0,04$ & $(-0,63 ; 0,66)$ & $-1,06$ & $(-1,39 ;-0,59)$ & $-0,07$ & $(-0,63 ; 0,66)$ \\
\hline $\mathrm{PC} / \mathrm{I}$, Fenton, percentil & 15,41 & $(8,19 ; 27,05)$ & 48,21 & $(26,42 ; 74,41)$ & 14,42 & $(8,19 ; 27,76)$ & 47,20 & $(26,42 ; 74,41)$ \\
\hline
\end{tabular}

Fonte: Dados da pesquisa.

Notas: PIG: pequeno para idade gestacional; AIG: adequado para idade gestacional; PC (perímetro cefálico); P/I (peso para idade); PC/I (perímetro cefálico para idade); " $p<0,05$ (Teste da soma dos postos sinalizados de Wilcoxon) - comparação entre os grupos PIG por Intergrowth e PIG por Fenton.

Tabela 2 - Características antropométricas no momento da alta/ transferência de lactentes nascidos PIG e AIG de acordo com os parâmetros de Intergrowth e Fenton.

\begin{tabular}{|c|c|c|c|c|c|c|c|c|}
\hline & \multicolumn{4}{|c|}{ Intergrowth } & \multicolumn{4}{|c|}{ Fenton } \\
\hline & \multicolumn{2}{|c|}{ PIG } & \multicolumn{2}{|c|}{ AIG } & \multicolumn{2}{|c|}{ PIG } & \multicolumn{2}{|r|}{ AIG } \\
\hline & Mediana & P25-75 & Mediana & P25-75 & Mediana & P25-75 & Mediana & P25-75 \\
\hline Idade, dias & 47,00 & $(13,00 ; 73,00)$ & 37,00 & $(22,00 ; 65,00)$ & 45,00 & $(11,00 ; 69,00)$ & 40,00 & $(22,00 ; 70,00)$ \\
\hline Peso, $\mathrm{g}$ & 2098,00 & $\begin{array}{r}(1890,00 \\
2380,00)\end{array}$ & 2231,00 & $\begin{array}{r}(2046,00 \\
2589,00)\end{array}$ & 2068,00 & $\begin{array}{r}(1877,00 ; \\
2189,00)\end{array}$ & 2238,00 & $\begin{array}{r}(2048,00 ; \\
2604,00)\end{array}$ \\
\hline $\mathrm{PC}, \mathrm{cm}$ & 33,50 & $(32,00 ; 34,00)$ & 32,00 & $(32,00 ; 34,00)$ & 33,00 & $(32,00 ; 34,00)$ & 32,00 & $(32,00 ; 34,00)$ \\
\hline $\mathrm{P} / \mathrm{I}$, Intergrowth, escore Z & $-2,87$ & $(-3,92 ;-2,12)$ & $-1,38$ & $(-2,16 ;-0,64)$ & $-2,87$ & $(-4,09 ;-2,10)$ & $-1,38$ & $(-2,19 ;-0,59)$ \\
\hline $\mathrm{P} / \mathrm{I}$, Intergrowth, percentil & 0,20 & $(0,00 ; 1,7)$ & 8,4 & $(1,50 ; 26,10)$ & 0,20 & $(0,00 ; 1,80)$ & 8,40 & $(1,40 ; 27,70)$ \\
\hline PC/I, Intergrowth, escore Z & $-1,76$ & $(-2,45 ;-1,10)$ & $-0,87$ & $(-2,12 ; 0,28)$ & $-1,76$ & $(-2,31 ;-1,18)$ & $-0,89$ & $(-2,12 ;-0,09)$ \\
\hline $\mathrm{PC} / \mathrm{I}$, Intergrowth, percentil & 7,60 & $(1,50 ; 22,10)$ & 30,10 & $(2,70 ; 77,30)$ & 7,60 & $(1,60 ; 33,90)$ & 28,3 & $(2,60 ; 77,40)$ \\
\hline $\mathrm{P} / \mathrm{I}$, Fenton, escore $\mathrm{Z}$ & $-2,92$ & $(-3,67 ;-2,30)$ & $-1,72$ & $(-2,29 ;-1,13)$ & $-2,96$ & $(-3,89 ;-2,26)$ & $-1,72$ & $(-2,29 ;-1,13)$ \\
\hline $\mathrm{P} / \mathrm{I}$, Fenton, percentil & 0,18 & $(0,02 ; 1,07)$ & 4,29 & $(1,12 ; 12,99)$ & 0,16 & $(0,01 ; 1,21)$ & 4,29 & $(1,12 ; 12,99)$ \\
\hline $\mathrm{PC} / \mathrm{I}$, Fenton, escore Z & $-1,18$ & $(-1,86 ;-1,01)$ & $-0,83$ & $(-1,54 ; 0,07)$ & $-1,24$ & $(-1,95 ;-1,01)$ & $-0,88$ & $(-1,54 ;-0,07)$ \\
\hline $\mathrm{PC} / \mathrm{I}$, Fenton, percentil & 11,94 & $(3,20 ; 15,56)$ & 20,36 & $(6,21 ; 47,20)$ & 10,69 & $(2,58 ; 15,56)$ & 18,87 & $(6,21 ; 47,20)$ \\
\hline
\end{tabular}

Fonte: dados da pesquisa.

Notas: PIG: pequeno para idade gestacional; AIG: adequado para idade gestacional; PC (perímetro cefálico); P/I (peso para idade); PC/I (perímetro cefálico para idade); ${ }^{p} p<0,05$ (Teste da soma dos postos sinalizados de Wilcoxon) - comparação entre os grupos PIG por Intergrowth e PIG por Fenton.

\section{DISCUSSÃO}

A comparação dos indicadores ao nascer, de acordo com os parâmetros de Fenton e Intergrowth, mostrou pequena diferença entre eles, com maior número de crianças diagnosticadas como PIG pela curva do Intergrowth $(25,5 \%$ x $27,7 \%)$, porém sem diferença estatística entre as proporções. Já no momento da alta/transferência, o que se observa é o contrário, com maior prevalência de PIG, considerado aqui como um dos possíveis diagnósticos de
RCEU, de acordo com as curvas de Fenton, com diferença estatística entre os grupos ( $73,6 \%$ por Fenton versus $64,9 \%$ por Intergrowth).

Achado semelhante foi encontrado em outro estudo (LEBRÃO; SUANO-SOUZA; SARNI, 2020) conduzido no Brasil, que avaliou 173 PT, com idades entre 26 e 33 semanas, durante o primeiro ano de vida; tal estudo observou ainda que o Intergrowth identificou 39,2\% nascimentos de crianças PIG versus $35,3 \%$ diagnosticadas por Fenton. 
A literatura parece apresentar alguma regularidade em relação aos achados a respeito da avaliação ao nascimento, de modo que, muitos estudos, inclusive envolvendo países de médio e baixo desenvolvimento, têm mostrado maiores percentuais de nascimento de crianças PIG, quando da utilização do referencial do Intergrowth (LEE et al., 2017; TUZUN et al., 2018).

Reddy et al. (2021) também obtiveram achado similar em estudo com 603 pré-termos nascidos com menos de 32 semanas gestacionais na Índia. Observou-se que a incidência de PIG (associado à ocorrência de RCIU) ao nascer foi maior quando da utilização dos gráficos de referência do Intergrowth, enquanto o RCEU na alta (peso para idade < P10) foi maior quando a referência utilizada foi o gráfico de Fenton.

O impacto clínico desses diferentes achados vem sendo estudado em profundidade, uma vez que é de grande importância para a assistência do paciente, seja no ambiente intra ou extra-hospitalar, a determinação de fatores de risco para desfechos tanto em curto quanto em longo prazo, os quais podem impactar na saúde futura do prematuro (REDDY et al., 2021; TUZUN et al., 2018). Porém, a literatura ainda apresenta achados divergentes a esse respeito, revelando que talvez outras variáveis, ainda não devidamente avaliadas, podem estar por trás das diferentes classificações observadas quando da aplicação desses referenciais.

Isso pode ser observado nos estudos de Reddy et al. (2021) e Tuzun et al. (2018), em que casos de nascimento PIG identificados apenas por Intergrowth, mas não por Fenton, tiveram risco aumentado de sepse e retinopatia da prematuridade (ROP), em comparação com os AIG no primeiro trabalho, e não apresentaram diferença no risco para morbidades a curto prazo, de acordo com o segundo estudo.

As diferenças encontradas para o padrão de crescimento pós-natal, de acordo com Fenton x Intergrowth, estão de acordo com o esperado e podem ser explicadas por meio da compreensão das diferentes metodologias aplicadas para a construção dessas duas curvas (CORDOVA; BELFORT, 2020). A curva de Fenton reflete dados do crescimento intrauterino de bebês, uma vez que compilou, por meio de uma metanálise, desfechos obstétricos (peso, comprimento e perímetro cefálico ao nascer) de quase 4 milhões de RN, ou seja, ela tem como alvo o percentil de nascimento do bebê e vem sendo amplamente utilizada como ferramenta de monitoramento do crescimento extrauterino de prematuros (TUZUN et al., 2018). Já o projeto Intergrowth 21 foi pensado com o objetivo de produzir padrões prescritivos para o crescimento entre a gravidez e a primeira infância, portanto foi desenvolvido a partir de um estudo prospectivo que produziu padrões de crescimento para o crescimento fetal (PAPAGEORGHIOU et al., 2014), crescimento pós-natal em prematuros (VILLAR et al., 2015) e padrões para atribuição de tamanho para idade gestacional no nascimento (VILLAR et al., 2014, 2016). Além disso, é válido ressaltar o cuidado metodológico em relação à escolha de oito locais geográficos para compor o estudo, o que garante a representatividade global dessa curva, além de todo o rigor em relação à realização das medidas antropométricas, dentre outras questões que conferem força a essa ferramenta.

Portanto, dadas todas as diferenças entre os ambientes intra e extrauterinos, ressaltando-se aqui a dificuldade no manejo clínico e nutricional do RN prematuro, é esperado que as curvas de padrões pós-natais de crescimento apresentem indicadores menores do que as curvas de crescimento intrauterino (CORDOVA; BELFORT, 2020). Maiores diferenças são observadas para os nascidos em idades gestacionais mais baixas (CORDOVA; BELFORT, 2020; MABHANDI; RAMDIN; BALLOT, 2019).

Isso justifica o menor número de lactentes diagnosticados com RCEU por meio da curva de Intergrowth na alta, em comparação a de Fenton. Ressalta-se aqui a importância do conhecimento acerca de cada uma das ferramentas e das referências utilizadas na prática clínica para avaliação nutricional, uma vez que isso influenciará no resultado das avaliações de crescimento e, consequentemente, na determinação da conduta nutricional para o paciente avaliado. A determinação do percentil de nascimento de um bebê como meta para seu crescimento pode ser potencialmente prejudicial na medida em que, para o atendimento dessa meta, será necessário garantir aumento da oferta nutricional para alcance de maior e mais rápido ganho ponderal, o que, por sua vez, pode estar associado a consequências metabólicas desfavoráveis a longo prazo (AMERICAN ACADEMY OF PEDIATRICS COMMITTEE ON NUTRITION, 1985).

Apesar da superioridade metodológica da curva do Intergrowth, muitos profissionais têm apresentado resistência em sua utilização. Tal fato se deve, em maior instância, a uma limitação desse estudo, expressa pelo pequeno número de bebês nascidos antes de 33 semanas de gestação, incluídos na amostra para a elaboração das curvas, o que limita sua validade (CORDOVA; BELFORT, 2020).

Análise dos indicadores ao nascer realizada apenas com os menores de 33 semanas apresentou resultados semelhantes aos encontrados para toda a população pesquisada. Tal resultado sugere que, para a população em questão, a ocorrência de RCEU parece não ter sido influenciada pela menor idade gestacional, fator que ainda tem sido utilizado como argumento contra a utilização da curva de seguimento do PT proposta pelo Intergrowth.

Outra forma de determinação do RCEU considerada no presente estudo foi a análise longitudinal dos indicadores de crescimento, caracterizando essa condição quando o traçado da curva era descendente e o delta entre indicadores do nascimento e do momento da alta/ transferência maior que um. Observou-se um menor número total de diagnósticos nos grupos, em comparação com a determinação transversal do RCEU (indicador de dado momento < P10). De acordo com essa classificação, a curva de Fenton detectou mais retardo do crescimento que a do Intergrowth $(64,70,3 \%$ por Fenton versus 51, $64,2 \%$ por Intergrowth), dessa vez, com uma diferença 
numericamente mais expressiva e estatisticamente significante.

Diretrizes recentes têm recomendado o uso da alteração do escore Z no peso, comprimento e perímetro cefálico como indicadores primários para identificar e documentar a desnutrição neonatal (GOLDBERG et al., 2018). A avaliação das trajetórias de crescimento revela mudanças no tamanho entre dois pontos no tempo (por exemplo, nascimento e alta da UTIN) e tem melhor capacidade preditiva para resultados de longo prazo do que avaliações transversais de tamanho (SHAH et al., 2006; SIMON et al., 2018).

Quando da utilização dessa forma de classificação da falha de crescimento pós-natal, é importante selecionar um ponto de corte sob o qual o declínio no escore $Z$ desde o nascimento seja considerado "anormal". De acordo com as trajetórias individualizadas de crescimento, declínios no escore $Z$ de peso para a idade maiores que 0,8 DP desde o nascimento são considerados anormais (CORDOVA; BELFORT, 2020).

Apesar das diferenças entre a determinação do RCEU longitudinal e transversamente descritas na literatura, foram observados percentuais muito próximos entre essas classificações: Intergrowth diagnosticou $64,9 \%$ de RCEU transversalmente e $64,2 \%$, longitudinalmente, enquanto Fenton diagnosticou $73,6 \%$ de RCEU transversalmente e $70,3 \%$, longitudinalmente.

Foram encontrados elevados percentuais de RCEU independente da forma de avaliação, o que deve servir de alerta para as unidades neonatais avaliadas, uma vez que essa condição é reflexo do estado nutricional do paciente e, consequentemente, da qualidade da assistência como um todo, mas especialmente da assistência nutricional neonatal ofertada (MAAS; POETS; FRANZ, 2015). Contudo, é importante ressaltar que os dados sobre terapia nutricional revelaram início precoce da NP e NE para mais de $70 \%$ e $60 \%$ da população, respectivamente. Há de se considerar ainda que os dados sobre oferta calórica e proteica ao longo do internamento não foram objetivo do instrumento de coleta de dados da Qualineo, bem como o alcance de metas nutricionais.

Questão importante ainda sobre a assistência nutricional das unidades avaliadas refere-se a não utilização de aditivos de leite humano, responsáveis por maior incremento ponderal durante o internamento especialmente de PT com muito baixo peso ao nascer (BROWN et al., 2016).

As taxas de infecção precoce e tardia observadas (42,6 e $21,3 \%$ ) estão abaixo das médias registradas pela Rede Brasileira de Pesquisas Neonatais em seu último relatório anual, divulgado em 2016 (NEONATAIS, 2016). Portanto, apesar da sabida influência dos processos infecciosos no estado nutricional do PT, possivelmente esse não foi um dos fatores associados aos percentuais elevados de RCEU observados (FREITAS et al., 2011).

Contudo, o uso de antibióticos registrado $(35,1 \%$ nas primeiras 48 horas de vida e 19,1\% até o 70 dia de vida) pode estar relacionado com a tolerância à dieta e, por conseguinte, ao alcance da NE plena de forma tardia, o que pode comprometer o estado nutricional dos PT durante o internamento (MARTINEZ et al., 2017).

Outra questão comumente associada ao risco de comprometimento do estado nutricional do PT, muito observada em internamentos prolongados, é a necessidade de suporte ventilatório por longos períodos, o que pode vir a caracterizar o quadro de broncodisplasia do PT. Esse diagnóstico pode ser considerado um fator de preocupação em termos nutricionais, tendo em vista o aumento do gasto energético provocado por essa patologia, além da restrição hídrica que ela impõe e que muitas vezes impede a oferta nutricional adequada ao paciente (MONTE et al., 2005). No presente estudo, a mediana de tempo de uso de oxigênio foi de 23 dias $(1 ; 18)$, abaixo do ponto de corte para diagnóstico de broncodisplasia (28 dias), no entanto, apenas 43 pacientes foram considerados para esta análise.

\section{CONCLUSÃO}

O diagnóstico de RCEU apresentou diferenças entre os dois referenciais utilizados na população avaliada, alcançando valores elevados ao final do internamento, de acordo com ambas as curvas. Os parâmetros de Fenton determinaram maior ocorrência de RCEU no momento do desfecho, independente da realização da avaliação transversal ou longitudinal dos indicadores. Tais diferenças possivelmente se devem a questões metodológicas próprias de cada uma das curvas, entretanto, não descreditam a utilização da curva do Intergrowth, recomendada atualmente pela Organização Mundial de Saúde para avaliação do crescimento pós-natal de PT.

Os elevados percentuais de nascimento PIG e da ocorrência de RCEU, ao longo do internamento, possivelmente estão associados ao perfil de pacientes comumente assistidos na unidade em questão, (1) em decorrência de gestações muitas vezes desassistidas e, em parte, (2) por serem provenientes de outras unidades; esta admissão de forma tardia faz com que cheguem graves e com comprometimento do estado nutricional, o que evidencia a necessidade da garantia ao nascimento em unidade terciária. Além disso há de se considerar a possibilidade de influência de fatores relacionados à assistência, que ainda demanda observações e melhorias.

\section{REFERÊNCIAS}

AMERICAN ACADEMY OF PEDIATRICS COMMITTEE ON NUTRITION. Nutritional needs of low-birth-weight infants. Pediatrics, Evanston, v. 75, n. 5, p. 976-986, May 1985.

BROWN, J. V. E. et al. Multi-nutrient fortification of human milk for preterm infants. The Cochrane Database of Systematic Reviews, Oxford, n. 5, p. CD000343, May 2016.

CLEMINSON, J. ; ZALEWSKI, S.; EMBLETON, N. D. What growth should we aim for in preterm neonates? Symposium: Neonatology, [s.l.], v. 27, n. 1, p. 18-22, 2017. Disponível em: https://www. paediatricsandchildhealthjournal.co.uk/article/S1751-7222(16)30176-7/ fulltext. Acesso em: 25 ago. 2021. 
CORDOVA, E. G.; BELFORT, M. B. Updates on assessment and monitoring of the postnatal growth of preterm infants. NeoReviews, Elk Grove Village, v. 21, n. 2, p. e98 LP-e108, Feb. 2020. Disponível em: http://neoreviews. aappublications.org/content/21/2/e98.abstract. Acesso em: 25 ago. 2021.

EHRENKRANZ, Richard A. Estimated fetal weights versus birth weights: should the reference intrauterine growth curves based on birth weights be retired? Archives of disease in childhood. Fetal and neonatal edition, London, v. 92, n. 3, p. F161-F162, May 2007. Disponível em: https:// pubmed.ncbi.nlm.nih.gov/17449851. Acesso em: 15 ago. 2021.

FENTON, T. R.; KIM, J. H. A systematic review and meta-analysis to revise the Fenton growth chart for preterm infants. BMC Pediatrics, London, $v$. 13, n. 1, p. 59, 2013. Disponível em: https://doi.org/10.1186/1471-243113-59. Acesso em: 15 set. 2021

FREITAS, B. A. C. de et al. Terapia nutricional e sepse neonatal. Revista Brasileira de Terapia Intensiva, Rio de jnaeiro, v. 23, n. 4, p. 492-498, dez. 2011. Disponível em: http://www.scielo.br/scielo.php?script=sci_ arttext\&pid=S0103-507X2011000400015\&lng=pt\&nrm=iso\&tlng=en. Acesso em: 30 ago. 2021.

GOLDBERG, D. L. et al. Identifying malnutrition in preterm and neonatal populations: Recommended indicators. Journal of the Academy of Nutrition and Dietetics, [s.I.], v. 118, n. 9, p. 1571-1582, Sept. 2018.

HENRIKSEN, C. et al. Growth and nutrient intake among very-low-birthweight infants fed fortified human milk during hospitalisation. The British journal of nutrition, [s.I.], v. 102, n. 8, p. 1179-1186, Oct. 2009.

LEBRÃO, C. W.; SUANO-SOUZA, F. I.; SARNI, R. O. S. Is the Intrauterine INTERGROWTH-21 growth curve better than fenton's for the classification at birth and prediction of postnatal growth in preterm infants? Maternal and Child Health Journal, New York, v. 24, n. 12, p. 1446-1453, 2020. Disponível em: https://doi.org/10.1007/s10995-020-02988-2. Acesso em: 30 ago. 2021

LEE, A. C. C. et al. Estimates of burden and consequences of infants born small for gestational age in low and middle income countries with INTERGROWTH-21st standard: analysis of CHERG datasets. BMJ, [s.I.], v. 358, 2017. Disponível em: https://www.bmj.com/content/358/bmj.j3677. Acesso em: 10 ago. 2021.

MAAS, C.; POETS, C. F.; FRANZ, A. R. Avoiding postnatal undernutrition of VLBW infants during neonatal intensive care: evidence and personal view in the absence of evidence. Archives of Disease in Childhood. Fetal and Neonatal Edition, London, v. 100, n. 1, p. F76-81, Jan. 2015.

MABHANDI, T.; RAMDIN, T.; BALLOT, D. E. Growth of extremely low birth weight infants at a tertiary hospital in a middle-income country. BMC Pediatrics, London, v. 19, n. 1, p. 231, 2019. Disponível em: https://doi. org/10.1186/s12887-019-1568-6. Acesso em: 21 ago. 2021.
MARTINEZ, F. E et al. Early empiric antibiotic use is associated with delayed feeding tolerance in preterm infants: A retrospective analysis. Journal of Pediatric Gastroenterology and Nutrition, New York, v. 65, n. 1, p. 107-110, July 2017.

MONTE, L. F. V. et al. [Bronchopulmonary dysplasia]. Jornal de Pediatria, Rio de Jnaeiro, v. 81, n. 2, p. 99-110, 2005.

NEONATAIS Rede Brasileira de Pesquisas. Relatório Anual 2016.[S.I: s.n.], 2016.

ONG, K. $\mathrm{K}$ et al. Postnatal growth in preterm infants and later health outcomes: a systematic review. Acta Paediatrica, Stockholm, v. 104, n. 10, p. 974-986, Oct., 2015. Disponível em: https://pubmed.ncbi.nlm.nih. gov/26179961. Acesso em: 01 set. 2021.

PAPAGEORGHIOU, A. T. et al. International standards for fetal growth based on serial ultrasound measurements: the fetal growth longitudinal study of the INTERGROWTH-21st Project. Lancet, London, v. 384, n. 9946, p. 869-879, Sept. 2014.

REDDY, K. V. et al. Comparison of Fenton 2013 growth curves and Intergrowth-21 growth standards to assess the incidence of intrauterine growth restriction and extrauterine growth restriction in preterm neonates $\leq 32$ weeks. The Journal of Maternal-fetal \& Neonatal Medicine, [s.I.], v. 34, n. 16, p. 2634-2641, ago. 2021.

SHAH, P. S. et al. Postnatal growth failure in preterm infants: ascertainment and relation to long-term outcome. Journal of Perinatal Medicine, Berlin, v. 34, n. 6, p. 484-489, 2006.

SIMON, L. et al. In Preterm Infants, Length Growth below Expected Growth during Hospital Stay Predicts Poor Neurodevelopment at 2 Years. Neonatology, Basel, v. 114, n. 2, p. 135-141, 2018.

TUZUN, F. et al. Comparison of INTERGROWTH-21 and Fenton growth standards to assess size at birth and extrauterine growth in very preterm infants. The Journal of Maternal-fetal \& Neonatal Medicine, [s.l.], v. 31, n. 17 , p. 2252-2257, Sept. 2018.

VILLAR, J. et al. International standards for newborn weight, length, and head circumference by gestational age and sex: the Newborn CrossSectional Study of the INTERGROWTH-21st Project. Lancet, London, v. 384, p. 857-868, 2014.

VILLAR, J. et al. INTERGROWTH-21st very preterm size at birth reference charts. Lancet, London, Feb. 2016

VILLAR, J. et al. Postnatal growth standards for preterm infants: the Preterm Postnatal Follow-up Study of the INTERGROWTH-21 st Project. The Lancet Global Health, [s.I.], v. 3, n. 11, p. e681-e691, 1 Nov. 2015. Disponível em: https://linkinghub.elsevier.com/retrieve/pii/ S2214109X15001631. Acesso em: 4 ago. 2021.

Submetido em: $19 / 11 / 2021$

Aceito em: 22/11/2021 